\title{
Capitalismo de plataformas: mi jefe es una App
}

\author{
Platform capitalism: my boss is an App
}

\section{Capitalismo de plataforma: meu chefe é um aplicativo}

\author{
Alejandra Dinegro Martínez ${ }^{1}$ \\ Universidad Nacional Mayor de San Marcos \\ alejandra.dinegro@unmsm.edu.pe \\ Código ORCID: https://orcid.org/0000-0002-2121-2964
}

\begin{abstract}
RESUMEN
Ante el desarrollo incesante de la tecnología y de la conectividad entre mercados, personas y empresas, ha surgido un nuevo modelo de hacer negocios. Algunos la denominan "gig economy", "sharing economy" y otros, simplemente, capitalismo de plataformas. Estos modelos de negocios están orientados a intermediar la demanda de servicios o bienes, de algunas personas, con otro sector de personas que ofrecen estos servicios o bienes de manera rápida, monitoreable y sencilla. Quienes intermedian esta conexión son plataformas digitales como Uber, Glovo, Rappi, Beat, Airbnb, UberEats, entre otras; las mismas que vienen asumiendo una interesante presencia en el Perú -en estos últimos cinco años- así como en América Latina y en varias partes del mundo. Al ser un modelo nuevo, presentan retos y desafíos, así como puntos ciegos que vienen siendo cuestionados al intentar responder algunas preguntas como ¿debe regularse la relación que se establece entre plataformas digitales y Apps con los taxistas o repartidores? O ¿Quién asume la responsabilidad si algo no sale bien? Este artículo describe el modelo de las apps de delivery, presentado como lo nuevo, y cómo viene introduciendo cambios en las relaciones laborales tradicionales al variar conceptos importantes como: contrato por "conexión", trabajador por "colaborador", horario de trabajo con "sé tu propio jefe", salario por "pago por servicio" o "despido por "bloqueo de la cuenta". Así mismo, comento la experiencia de mi breve investigación participante realizada en la ciudad de Lima y como este modelo de negocio reacciona: ¿si representa más un riesgo o una oportunidad, para las personas?
\end{abstract}

\begin{abstract}
Faced with the incessant development of technology and connectivity between markets, people and companies, a new model of doing business has emerged. Some call it "gig economy", "sharing economy" and others, simply, platform capitalism. These business models are aimed at intermediating the demand for services or goods, of some people, with another sector of people that offer these services or goods in a quick, monitoring and simple way. Those who mediate this connection are digital platforms such as Uber, Glovo, Rappi, Beat, Airbnb, UberEats, among others; the same ones that have been assuming an interesting presence in Peru -in the last five years- as well as in Latin America and in various parts of the world. Being a new model, they present challenges and challenges, as well as blind spots that have been questioned when trying to answer some questions such as: should the relationship established between digital platforms and Apps with taxi drivers or delivery drivers be regulated? ¿O who takes responsibility if something goes wrong? This article describes the delivery apps model, presented as new, and how it has been introducing changes in traditional labor
\end{abstract}

1 Posgrado de la Facultad de Ciencias Sociales. Bachiller en Sociología

Recibido: 04/10/2019 - Aceptado: 03/05/2020 - Publicado: 28/08/2020

Citar como:

Dinegro, A. (2020). Capitalismo de plataformas: mi jefe es una App. Espiral, revista de geografías y ciencias sociales, 2(3), 123 - 131. http://dx.doi.org/10.15381/espiral.v2i3.18452

C Los autores. Este artículo es publicado por Espiral, revista de geografías y ciencias sociales de la Universidad Nacional Mayor de San Marcos. Este es un artículo de acceso abierto, distribuido bajo los términos de la licencia Creative Commons Atribucion- No Comercia_Compartir Igual 4.0 Internacional. (http://creativecommons.org/licenses/by-nc-sa/4.0/) que permite el uso no comercial, distribución y reproducción en cualquier medio, siempre que la obra original sea debidamente citada. 
relations by varying important concepts such as: contract for "connection", worker for "collaborator", working hours with "I know your own boss", salary for "service payment" or "dismissal for "account blocking". Likewise, I comment on the experience of my brief participant research carried out in the city of Lima and how this business model reacts: ¿if it represents more of a risk or an opportunity, for people?

\section{RESUMO}

Diante do desenvolvimento incessante de tecnologia e conectividade entre mercados, pessoas e empresas, surge um novo modelo de fazer negócios. Alguns chamam de "economia de gig", "Economia da partilha" e outros, simplesmente, capitalismo de plataforma. Esses modelos de negocios destinamse a intermediar a procura de bens ou serviços, de algumas pessoas, com outro setor de pessoas que oferecem esses serviços ou bens de forma rápida, fácil e simples. Quem medeia essa conexão são plataformas digitais como Uber, Glovo, Rappi, Beat, Airbnb, UberEats, entre outras; os mesmos que vêm assumindo uma presença interessante no Peru - nos últimos cinco anos - bem como na América Latina e em várias partes do mundo. Por se tratar de um novo modelo, apresentam desafios, bem como pontos cegos que têm sido questionados ao tentar responder a algumas questões como, por exemplo, deve ser regulamentada a relação que se estabelece entre plataformas digitais e Apps com taxistas ou entregadores? Ou quem assume a responsabilidade se algo der errado? Este artigo descreve o modelo de app de entrega, apresentado como o novo, e como ele vem introduzindo mudanças nas relações de trabalho tradicionais, variando conceitos importantes como: contrato por "conexão", trabalhador por "colaborador", horário de trabalho com "saber seu próprio patrão ", salário por" taxa de serviço "ou" demissão por "bloqueio de conta". Da mesma forma, comento a experiência de minha breve pesquisa participante realizada na cidade de Lima e como esse modelo de negócio reage: se representa mais um risco ou uma oportunidade para as pessoas?

PALABRAS CLAVE: economía colaborativa; plataformas digitales; relaciones laborales; tecnología; Cuarta Revolución Industrial.

KEYWORDS: collaborative economy; digital platforms; labor relations; technology; Fourth Industrial Revolution.

PALAVRAS-CHAVE: economia colaborativa; plataformas digitais; relações de trabalho; tecnologia; Quarta Revolução Industrial.

\section{Introducción: ¿economía colaborativa o capitalismo de plataformas?}

Alrededor de lo que se conoce como plataformas digitales, viene sucediendo un fenómeno económico, laboral y tecnológico que cuenta con apenas diez años de existencia a nivel mundial, según lo menciona el informe "Economía de Plataformas y empleo: ¿Cómo es trabajar para una app en Argentina? (2019). Y viene significando uno de los sucesos disruptivos para el mundo del trabajo, a nivel mundial y por supuesto, no es ajeno al Perú.

Para poder entender de lo que se está exponiendo en el presente texto, considero importante partir por entender conceptos básicos que se le atribuyen a esta transformación de las relaciones laborales tradicionales que van de la mano con la aparición de nuevas innovaciones tecnológicas.

El trabajo en plataformas digitales surgió a principios de los años 2000 con el crecimiento de la Internet y la necesidad de contar con participación humana en las tareas necesarias para el buen funcionamiento de las industrias en línea. Los trabajadores pueden trabajar desde cualquier lugar del mundo en tanto tengan una conexión a Internet. Los puestos de trabajo van desde la sofisticada programación informática, el análisis de datos y el diseño gráfico, hasta las relativamente sencillas "microtareas" de naturaleza administrativa. (OIT, 2019)

Por un lado, tenemos al espíritu empresarial que afirma que hoy en día vivimos en una era de profunda transformación y que, de la mano de la tecnología, ésta puede facilitarnos la vida en las más mínimas tareas que consumen nuestro tiempo (pedir un delivery, tramitar un documento, pedir un taxi, buscar un hospedaje, entre otros.). Y para lograr ello, desde el campo tecnológico han aparecido diversas plataformas 
digitales (principalmente, de servicios) que han aparecido como la promesa de satisfacción del más mínimo de nuestros antojos. Desde el campo de la economía, diversos conceptos intentan describir estas infraestructuras digitales, como "economía colaborativa", "sharing economy" o "gig economy".

En el informe "Economía colaborativa para América Latina", publicado por el Banco Interamericano de Desarrollo (BID), en el 2016, los principales CEOS de empresas digitales españolas, describen a la economía colaborativa como un presente y futuro prometedor para América Latina y el Caribe, así como una oportunidad para insertarnos a la Cuarta Revolución Industrial, como se discutió en el Foro Económico Mundial, en Davos, Suiza, en el año 2016. Afirman que este nuevo paradigma ofrece importantes oportunidades para favorecer la inclusión social, promover el espíritu emprendedor y desencadenar una ola de innovación que pueda contribuir a resolver algunos de los grandes problemas sociales, económicos y ambientales de los habitantes de la región. (BID, 2016). O al menos eso es lo que manifiestan.

Por otra parte, el profesor de Economía Digital del Departamento de Humanidades Digitales del King's College en Londres, doctor Nick Srniceck, se refiere a este campo de la economía como el "Capitalismo de Plataformas", título de su libro presentado en el año 2018. El argumento de este libro es que, con una prolongada caída de la rentabilidad de la manufactura, el capitalismo se volcó a los datos como un modo de mantener el crecimiento económico y la vitalidad de cara al inerte sector de la producción. (Srniceck, 2018).

Dado que la economía digital y al interior de ella se expande cada vez más estas caracterizaciones de empresas cuyos medios de producción son las infraestructuras digitales capaces de organizar nuestras vidas, ciudades, negocios y hasta gobiernos; es importante mencionar que adquieren y administran una infinita cantidad de datos. Los mismos que se vuelven rentables para mantener la relación con usuarios, (clientes), trabajadores (colaboradores) y plataforma digital.

Un importante informe de la Organización Internacional del Trabajo (OIT), publicado en el 2019, menciona lo siguiente:

"Pese a que resulta algo muy valioso para varias compañías exitosas, el trabajo en las plataformas digitales de microtareas suele caracterizarse por remuneraciones inferiores al salario mínimo, flujos impredecibles de ingresos y la ausencia de protecciones laborales que suelen observarse en una relación de trabajo típica. No obstante, ninguno de estos resultados negativos es inherente a esta modalidad de trabajo o a las microtareas. Por el contrario, es posible reconfigurar las modalidades de micro trabajo para mejorar las condiciones de los trabajadores." (OIT, 2019)

He aquí una de las principales diferencias conceptuales de fondo, que se pueden percibir entre ambas partes que definen este nuevo modelo de negocios. Pero, ¿sabemos cuáles son los elementos que le dan la razón de ser a estas plataformas y aplicativos móviles (apps)? ¿Qué tanto sabemos sobre ellas? ¿De qué tipo son las modalidades de trabajo que se ofrece a través de ellas? ¿Es colaboración o trabajo lo que se ofrece en estas plataformas? ¿Qué riesgos y oportunidades plantean para la sociedad en general? ¿Existen medidas regulatorias frente a este nuevo fenómeno comercial? ¿Cuáles son los cambios que vienen introduciendo en el mundo del trabajo peruano desde su funcionamiento? Este artículo se propone responder estas preguntas sobre la base de la información recolectada y la investigación participante realizada en la ciudad de Lima, durante el mes de agosto del año 2019.

\section{II. ¿Oportunidad o precariedad laboral?}

En la ciudad de Lima, en Perú, son cada vez son más las personas -especialmente jóvenes- los que usan estas plataformas digitales a través de aplicativos móviles, 
fácilmente descargables en el celular. Lo hacen para solicitar un servicio, pero también para asumir una tarea dentro de ella.

Las personas que opten por asumir una labor que le encomienda la app (a través de algoritmos), se caracterizan por tener bajos niveles de calificación para obtener un trabajo en un mercado laboral cada vez más competitivo y a su vez informal y precario. Inicialmente, no se requiere más que un celular, internet, auto, bicicleta o motocicleta. En el mejor de los casos, un seguro de salud, licencia de conducir, entre otros trámites administrativos que son asumidos por los "colaboradores". Y se puede empezar a desarrollar actividades dentro de las 48 horas de registrarse en la app o dependiendo del flujo de atención, hasta en una semana.

Tal y como lo describo en mi columna "Apps Capitalism", publicada en la revista The North American Congress on Latin America (2019): en estas plataformas no hay un sueldo o salario mínimo legal. De hecho, no hay ni contrato, seguro de salud, horario de trabajo o alguna modalidad de vínculo laboral. Las ganancias que obtiene un repartidor o conductor, dependen del porcentaje que aplican en cada país, incluyendo otros aspectos como la distancia, tiempo invertido durante las "horas punta" o las veces que el "colaborador" rechace o acepte las solicitudes.

UberEats, Rappi y Glovo, son las principales apps de delivery que operan en la ciudad de Lima y vienen produciendo importantes cambios en las relaciones laborales que, tradicionalmente, conocemos. Es un modelo económico en el que los trabajadores son considerados colaboradores y en algunos casos, trabajadores independientes, sin ningún tipo de compromiso legal, laboral, económico y social de por medio. Pues argumentan que solo son mediadoras que conectan colaboradores, servicio y usuarios. Argumentos que se pueden encontrar en la publicidad de estas Apps.

Una nueva modalidad laboral que se sostiene en una innovadora forma de crear trabajo, sin que éstos califiquen como tales y sin que, los que realicen las actividades, no terminen asumiéndose como tales: como trabajadores.

Sin embargo, la información vinculada a las formas de trabajo a través de estas plataformas digitales, de delivery, no ha logrado manifestarse en las estadísticas oficiales, ni ante las principales instituciones públicas del país, que tienen implicancia en material laboral. Por lo que esta forma de trabajo, queda invisibilizada en medio de otras categorías: ocupados no asalariados, trabajadores por cuenta propia, algunas formas de trabajo atípico asalariado o trabajadores informales e incluso, subempleo.

En el Perú, como en la región y en el mundo, la precarización laboral, el desempleo y la informalidad laboral resultan ser fenómenos mundiales que afectan mayormente a los jóvenes y constituyen fenómenos multidimensionales e intersectoriales. En Latinoamérica, el problema de la precariedad laboral es una problemática sin resolver y, por el contrario, las cifras de desempleo, informalidad, subempleo y autoempleo, se han intensificado de manera acelerada durante los últimos diez años, según el propio informe "Perspectivas sociales y del empleo en el mundo", publicado por la OIT, en el año 2018.

Según el reporte trimestral del Instituto Nacional de Estadística e Informática (INEI), la tasa de desempleo juvenil creció 7,2\% entre abril y junio de este año. Cifra más alta que las de otros grupos de edad. (Diario El Comercio, 2019, párr. 1).

En el Perú, como en el mundo, estas empresas despliegan subliminales y millonarias campañas publicitarias, que tiene como fin convencer a los jóvenes de ser colaboradores o emprendedores. El mensaje escondido de la economía colaborativa es volver, un producto, como servicio. Tal y como suelen explicarlo en los talleres de coaching digital. 
Estas plataformas digitales y apps, esconden condiciones laborales que bordean con las prácticas de esclavitud laboral e informalidad, pues se trabaja más de ocho horas diarias, son expuestos a calificaciones (que abre la posibilidad a la arbitrariedad de las mismas) y de acuerdo a ello, algorítmicamente, se les otorga ciertas cantidades de pedidos a atender. En caso de comenzar a rechazar los pedidos, el trabajador empieza a recibir menos solicitudes hasta que es suspendido de forma temporal o definitiva de la plataforma. Volver a reintegrarse puede ser una posibilidad, en otras ocasiones no. Entonces, ¿qué tipo de relación mantienen estas personas con las plataformas digitales y/o apps?

Las empresas manifiestan que no existe ningún tipo de vínculo contractual, pues son solo plataformas digitales puestas a disposición de las personas. Son intermediarias. Sin embargo, estas argumentaciones son desmentidas por la jurisprudencia internacional de Europa, especialmente. Dónde señalan que sí mantienen dependencia económica y que por lo tanto no son trabajadores autónomos ni independientes. Este punto, lo detallaremos más adelante.

La OIT, considera que estos modelos de empleo contribuyen a la precariedad laboral de los países donde estas prácticas están permitidas. Alerta que, de no atenderse esta problemática, las generaciones futuras estarán conformadas por jornaleros digitales, con consecuencias como poco acceso a la seguridad social, presión a la baja de los salarios mínimos, perpetuo riesgo de exclusión social, cambio en las relaciones entre trabajo y sociedad, afectando considerablemente los elementos de cohesión social, entre otros aspectos. (OIT, 2019).

Se ha mencionado que el trabajo en plataformas digitales se asemeja a muchas modalidades laborales de vieja historia, que en este caso agregan simplemente una herramienta digital como intermediaria. Las estrategias de trabajo que desagregan tareas en unidades pequeñas para asignarlas a trabajadores no calificados parecen ser "una regresión a los procesos industriales no calificados asociados con Taylor, pero sin la lealtad o la seguridad en el trabajo" (Cherry, 2016a, p. 3).

Va quedando claro que al día de hoy se están produciendo aceleradas transformaciones en las modalidades laborales y que trabajar en plataformas digitales podría comprenderse mejor como parte de un fenómeno mayor: la automatización del empleo. Comprender que el fenómeno de las plataformas digitales y apps, forman parte de un proceso mucho mayor, es clave para saber cómo enfrentarlo de la manera más óptima posible, donde la tecnología aporte un rol fundamental en el desarrollo del ser humano y la superación de procesos sociales desiguales e inequitativos.

\section{III. ¿Cómo es trabajar para una app en Lima?}

Entre setiembre y agosto del 2019, decidí a través de la metodología de la investigación participante, experimentar, cómo es un día de reparto a través de la aplicación móvil más usada en Lima: Glovo.

Para ello, en primer lugar, es importante mencionar, que los resultados obtenidos, son una pequeña muestra obtenida en las semanas de entrevistas, conversaciones vía celular y participación en reuniones realizadas en la calle. Resultados que se encuentran aún en camino a un procesamiento final, puesto que se asumió el compromiso de mantener las identidades en reserva para evitar algún inconveniente a los trabajadores que participaron: jóvenes ciudadanos venezolanos quienes, accedieron a brindar, fácilmente, la información y predisposición a grabar un reportaje posterior. El mismo que se realizó durante el mes de agosto del 2019 en los distritos de Miraflores, Surquillo y Cercado de Lima. Y se encuentra en el portal de YouTube con el nombre de "La mochila pesada de los trabajadores por aplicación, en Lima". 
En una columna publicada por El Diario El Comercio, se menciona que, hasta marzo del 2019, se tiene conocimiento que son más de 3.500 'Rappis' y 6.000 'Glovers' las cuentas activas que realizan repartos. En su mayoría hombres jóvenes y migrantes (a estos últimos se les suele alquilar la cuenta que un peruano crea). Ya que los filtros o requisitos para acceder a una plataforma son mínimos, "sacarle la vuelta" a la app es una de las consecuencias de un vacío regulatorio intrínseco y exterior.

El 17 de junio de este año, un trabajador de la empresa Glovo quedó gravemente herido tras haber sufrido un accidente vehicular en el distrito de La Molina. El repartidor, identificado como un ciudadano venezolano, perdió una pierna en pleno desarrollo de su actividad laboral. La empresa, a través de un comunicado, expresó su solidaridad con el repartidor y su disposición a colaborar con las autoridades para ofrecer toda la información registrada en su sistema, para la resolución del caso. Nada más. (Dinegro. IDL, párr. 16).

La ciudad de Lima, es una de las menos amigables para el manejo de vehículos menores. Si manejar bicicleta en la capital, es complicado, ¿Se imagina lo que es hacerlo mientras va cargando una caja de aluminio en la espalda y el tiempo a cuestas presionándolo por hacerlo rápido? Pues esa es la situación que decidí experimentar durante la grabación del reportaje mencionado.

Para ello decidí ponerme en contacto con algunos de los repartidores y fue como a través de una cuenta en Instagram, denominada "Glovers en Perú", conocí a Raúl. Gracias a él, pudimos acceder a grabar el reportaje y entrevistar a Andrea y Luis.

Así mismo, para recoger la opinión de los repartidores peruanos, fue el mismo modo de contacto, pero esta vez, a través de un fan page denominado "Confesiones de un glover", el cual es administrado por Marco y por su intermedio, accedimos también a entrevistar a Samuel.

A continuación, los participantes, características, nacionalidad, edad y aplicación móvil donde trabajan o trabajaron.

Tabla $\mathbf{N}^{\circ} 1$.

\begin{tabular}{ccccc}
\hline $\mathbf{N}^{\circ}$ & Nombre & Nacionalidad & Edad & App donde trabaja/trabajó* \\
\hline 1 & Raúl & Venezolana & 28 & Glovo \\
2 & Luis & Colombiana & 28 & Glovo \\
3 & Andrea & Venezolana & 30 & Glovo* \\
4 & Marco & Peruano & 28 & Glovo \\
5 & Samuel & Peruano & 24 & Glovo/UberEats y Rappi* \\
\hline
\end{tabular}

Elaboración propia. $^{2}$

Para los ciudadanos venezolanos, este tipo de aplicaciones representa una oportunidad ineludible de acceder a un tipo de ingresos, en un mercado laboral que les es ajeno y familiar. Sin embargo, como se constató, no se consideran colaboradores, sino trabajadores y, por lo tanto, con derechos laborales que deberían ejercer. Para este grupo de repartidores, éste sería su observación principal puesto que consideran que estas empresas se aprovechan de las necesidades de las personas y que no pueden reclamar ante un bloqueo de cuenta u otra actividad que atente contra lo que es, su principal fuente de ingresos.

Para los ciudadanos peruanos, esta labor, califica como una actividad que inicialmente, no desempeñaban en horarios extendidos, pero con el tiempo y ante la dificultad de encontrar otro tipo de empleo, optaron por convertir esta actividad en un trabajo

2 Los nombres mencionados, son ficticios. Por el compromiso asumido de no evidenciar sus nombres de pila para evitar algún tipo de represalia como el bloqueo de sus cuentas, etc. 
de tiempo completo. Coincidían con los ciudadanos venezolanos en no considerarse colaboradores, pero que ese sería un detalle secundario, lo principal para -ellos- era la seguridad y tener mayor cantidad de pedidos, lo cual es sinónimo de más ingresos.

Los cinco participantes coincidieron en mencionar que los que les convenció a optar por esta actividad fue la posibilidad de poder decidir sus propios horarios y de qué tareas aceptar y cual no. No obstante, todos afirmaron que trabajan en horarios no convencionales. Podían conectarse a la aplicación desde las 6am y acabar a las 12 de la medianoche. En efecto, es durante las noches y los fines de semana, que el requerimiento de servicios por parte de los consumidores, se convierten en horas punta de atención y repartos. En pocas palabras, son los horarios y días donde mayores ingresos pueden obtener.

Importante mencionar que mientras para Raúl, Luis y Andrea, esta actividad fue y es su principal actividad a la que se dedican -desde que se conectaron a la aplicaciónpara Marco y Samuel, fueron, inicialmente, una fuente de ingresos complementaria a sus actividades principales. Por lo tanto, no le dedicaban la mayor cantidad de horas de su día y ambos lo hacían en sus ratos libres. Posteriormente, se convirtió en su principal fuente de ingreso.

Con relación a las calificaciones y los pagos, todos señalaron que en algún momento afrontaron situaciones desagradables con la empresa y con los clientes. Señalaron que muchas veces un pedido era cancelado por el cliente o que algún cliente se había negado a pagar. En otras ocasiones, el cliente decidía otorgar calificaciones bajas al repartidor si este no dejaba el pedido en la puerta de la casa (a pesar de que la aplicación señala que el repartidor no debe subir escaleras o ascensores) o si este resultaba ser ciudadano extranjero. Como consecuencia de ello, las plataformas, a través de los algoritmos, evalúan el desempeño de los trabajadores, de manera unidireccional puesto que no hay mecanismos para evaluar de manera inversa.

Los cinco encuestados, manifestaron su frustración y rechazo por no poder tener derecho a réplica. Puesto que, en el peor de los casos, sufren el bloqueo de sus cuentas, sin previo aviso, como fue el caso de Andrea, y no se les comunica el porqué de ello. Importante mencionar que para los repartidores resulta complicado comunicarse con el área de soporte para manifestar estos inconvenientes y tampoco lo pueden hacer en alguna oficina física, ya que, en Lima, la oficina central cambia de dirección constantemente y todas las citas son con plazos de anticipación por medio del correo electrónico.

Muchos de ellos, recurren a otro tipo de canales para poder comunicarse, orientarse y ayudarse en alguna dificultad que se les presente en la aplicación, como grupos de WhatsApp, grupos de Facebook y Fan Page.

Finalmente, aspectos como la salud y seguridad en el trabajo, acoso, xenofobia e inseguridad ciudadana, son daños colaterales totalmente asumidos por los repartidores. Todos mencionaron que reasignan algunos pedidos porque ellos consideran que ponen en riesgo su integridad física y la de sus materiales de trabajo. Reasignar, significa trasladar una solicitud del servicio a otro trabajador que puede y desea realizarlo. Para la persona que reasigna una tarea, puede costarle, con el tiempo, una baja en sus calificaciones. Por lo tanto, menor asignaciones de pedidos, en consecuencia, menos ingresos y finalmente, el bloqueo de su cuenta.

\section{Jurisprudencia e intentos de regulación a las apps}

A pesar de explorar un escenario favorable para los ingresos de estas plataformas digitales y aplicaciones móviles, sigue habiendo espacios oscuros que aún no han podido responder. Es así que vienen afrontando batallas legales, juicios, sanciones económicas, regulaciones estatales y la prohibición de ingresar a algunos países. 
A continuación, algunos hitos importantes:

- En el 2013, Uber, perdió un juicio interpuesto por tres es conductores de la ciudad de San Francisco, Estados Unidos. Solicitaron que se les reconozca como trabajadores.

- $\quad$ En el 2017, Uber vuelve a perder un juicio ante el Tribunal de Justicia de la Unión Europea que sentencia que es un servicio de transporte y no una plataforma digital colaborativa ${ }^{3}$. Se sumaron protestas en San Francisco, exigiendo aumento en las tarifas de pago.

- En el 2018, Glovo afrontó perdió contra tres juicios demandados en Madrid, al reconocer la existencia de un vínculo laboral entre empresa y los mal llamados colaboradores. Se sumaron protestas en Valladolid, Zaragoza, Madrid y Barcelona.

En agosto de 2019, el estado de California en Estados Unidos, ha aprobado una ley dirigida a reducir drásticamente el número de falsos autónomos en la economía de plataformas.

\section{Propuestas para su regulación: alternativas en América Latina}

Resulta curioso cómo, en Europa, se van sentando precedentes jurídicos con relación a la regulación de estas plataformas digitales, que van acompañadas de la auto-organización de los trabajadores, a diferencia de América Latina, donde la organización es casi incipiente pero que, en respuesta, son iniciativas legislativas las que vienen incidiendo en algún tipo de regulación. Veamos.

Tabla $\mathbf{N}^{\circ} 2$.

\begin{tabular}{|c|c|c|c|c|}
\hline $\mathbf{N}^{\circ}$ & País & Iniciativa & Año & Descripción \\
\hline 1 & Costa Rica & $\begin{array}{l}\text { Proyecto de Ley } \\
\mathrm{N}^{\circ} 21.228\end{array}$ & 2018 & $\begin{array}{l}\text { Proyecto de reforma al sistema de transporte remunerado de } \\
\text { personas y regulación de las Empresas de Plataformas Tecnológicas } \\
\text { de transporte. Autor: Presidente Carlos Alvarado Quesada }{ }^{4} \text {. }\end{array}$ \\
\hline 2 & Argentina & $\begin{array}{l}\text { Asociación de Personal } \\
\text { de Plataformas (APP) }\end{array}$ & 2018 & $\begin{array}{l}\text { Sindicato que busca organizar a los trabajadores que transportan } \\
\text { productos y personas en el marco de las nuevas plataformas digitales. }\end{array}$ \\
\hline 3 & Chile & $\begin{array}{l}\text { Proyecto de Ley, } \\
\mathrm{N}^{\circ} 12475-13\end{array}$ & 2018 & $\begin{array}{l}\text { Proyecto de ley sobre los derechos de las trabajadoras y trabajadores } \\
\text { de Plataformas Digitales de Servicios. Autor: Diputado Gabriel Boric }{ }^{5} \text {. }\end{array}$ \\
\hline 4 & Perú & $\begin{array}{l}\text { Proyecto de Ley } \\
\mathrm{N}^{\circ} 4243\end{array}$ & 2019 & $\begin{array}{l}\text { Ley del empleo digno que regula a los trabajadores de plataformas } \\
\text { digitales. Autor: Ex Congresista Manuel Dammert y Alejandra } \\
\text { Dinegro. }{ }^{6}\end{array}$ \\
\hline
\end{tabular}

Elaboración propia.

A modo de conclusiones:

- El debate sobre el futuro del trabajo y en especial el, trabajo en condiciones decentes, está cobrando creciente interés a nivel global. Es importante que, desde el Derecho, se puedan crear mecanismos vanguardistas que no permitan tener vacíos legales en materia de derecho laboral, así como fiscales o administrativos.

- Se requiere un nuevo enfoque en la inspección y fiscalización laboral que vaya de lo prevención a lo sancionador y no en viceversa.

- Se requiere crear u optimizar herramientas de análisis, registro y contabilidad de las personas que trabajan en estas aplicaciones móviles.

3 Sentencia del Tribunal de Justicia de la Unión Europea (Gran Sala) del 20 de diciembre de 2017.

4_https://www.nacion.com/el-pais/servicios/carlos-alvarado-anuncia-proyecto-para-que-uber-y/Y7YJUF2X6JB6ZCIE-

BRK2BL4E4Y/story/

5 https://web.mijefeesunaapp.cl/cms/wp-content/uploads/2019/03/Proyecto-de-Ley-mi-jefe-es-una-App.pdf

6 http://www2.congreso.gob.pe/Sicr/TraDocEstProc/CLProLey2016.nsf/Parlamentarios/A35385A242D-

21C64052583E4005A056 
- Se requiere tener un Plan de transición en materia educativa que otorgue cualificaciones acordes a los cambios tecnológicos, a las generaciones que tiene menos posibilidades de insertarse a una era digital.

- Promover el diálogo social, fortaleciendo los sindicatos y toda forma de organización no tradicional de estos trabajadores.

- Para el caso peruano, se requiere contar con una Ley General del Trabajo que permita construir un piso común de derechos laborales para todo trabajador (de cualquier edad, sexo, raza, función, actividad productiva o modalidad.

- La regulación del empleo que generan las plataformas digitales de delivery es tan necesario como la regulación del trabajo independiente o autónomo el cual se desarrolla en situaciones altas de informalidad.

El mundo del trabajo y su evolución merece una atención especial, pues se trata de personas intercambiando su libertad por un pago correspondiente, y su protección no debería quedar relegada a la posibilidad que cada uno de ellos, pueda cubrir.

Por ello es importante que, frente a crisis económicas, sanitarias y de vulneración de derechos que puedan suscitarse, las propuestas por mejorar las condiciones laborales de los repartidores, debe continuar. Es fundamental que se tome consciencia y, como estados, nos hagamos cargo de lo que producimos, de lo que consumimos y de las condiciones en las que nos relacionamos con los demás, y evitar que la desigualdad y la injusticia social siga sosteniéndose sobre situaciones de vidas precarias y por el contrario se garanticen condiciones de trabajo digno para todos.

\section{Referencias Bibliográficas}

BID. (2016). "Economía Colaborativa en América Latina" Recuperado de: https://publications. iadb.org/es/

Dinegro, A. (2019). App Capitalism. NACLA Report on the Americas, 51(3), 236-241. doi: 10.1080/10714839.2019.1650485.

Dinegro, A. (2019). Plataformas y empleo: ¿cómo es trabajar para una app en el Perú? Ideele, (286). Recuperado de: https://revistaideele.com

Dinegro, A. (2019). La mochila pesada de los trabajadores por aplicación, en Lima. [Reportaje]. Disponible en: https://www.youtube.com

InfoCuria Jurisprudencia. (2017). Sentencia del Tribunal de Justicia de la Unión Europea. Recuperado de: http://curia.europa.eu/

Inga, C. (30 de marzo de 2019). Ganancias y empleo en la era de las apps de delivery: ¿cómo ganan los repartidores? El Comercio. Recuperado de: https://elcomercio.pe

Madariaga, J., Buenadicha, C., Molina, E. y Ernst, C. (2019). Economía de plataformas y empleo ¿Cómo es trabajar para una app en Argentina? Recuperado de: https://www.cippec.org/

OIT. (2018). Perspectivas sociales y del empleo en el mundo. Recuperado de: https://www.ilo.org/

OIT. (2019). Las plataformas digitales y el futuro del trabajo. Cómo fomentar el trabajo decente en el mundo digital. Recuperado de: https://www.ilo.org/

OIT. (2019). Trabajar para un futuro más prometedor. Recuperado de: https://www.ilo.org/

Redacción EC. (16 de julio de 2019). Lima: Empleo adecuado entre jóvenes se reduce en 7,9\% entre abril y junio. El Comercio. Recuperado de: https://elcomercio.pe

Srnicek, N. (2018). Capitalismo de plataformas. Buenos Aires, Argentina: Editorial Caja Negra. 
